\title{
Variable Step Size LMS Algorithm Based on Cosine Function
}

\author{
ZHANG Jingjing \\ Army Academy of Armored Forces, Beijing, 100072, China \\ zhanglingrui007@163.com
}

Keywords: variable step size LMS algorithm; weak signal; cosine function; convergence rate; noise cancellation

Abstract. In this paper, a new variable step size LMS algorithm based on cosine function is proposed. The weak signal is difficult to extract, so we constructed a new relationship between the step size and the error signal, which depend on the mathematical transformation of the cosine function, and also analyzed the parameter values which effects the performance of the algorithm mainly. In the noise cancellation system, the performance of the algorithm is simulation by MATLAB software. The result shows that the algorithm has better effect during signal denoising, especially in the weak signal extraction which with low SNR(signal to noise ratio), filtering effect is better.

\section{Introduction}

Adaptive filtering is an important part in the field of statistical signal processing. The fixed step size algorithm cannot process effectively for the signal which statistical information is unknown. But the variable step size algorithm can update the internal parameters automatically to follow the changes of external environment, so it becomes a hot spot in the field of signal processing. Noise elimination is an important application of adaptive algorithm. In adaptive cancellation system, the commonly used algorithms are the LMS (Least-mean-square) algorithm and RLS (Recursive-Least-square) algorithm. The LMS algorithm is widely used because of its simple structure, low computational complexity and easy implementation. It based on the steepest descent method, to reach the optimal value of objective function, the step size factor updates along the negative direction of the gradient vector valuations of the weight coefficient, so as to realize the adaptive filter in sense of square $\operatorname{error}^{[1]}$.

\section{The LMS Algorithm}

The schematic diagram of adaptive filter is shown in figure 1. $\mathrm{X}(\mathrm{n})$ is the input signal vector of adaptive filter at $n$ times, $V(n)$ is the noise signal, $y(n)$ is the output signal, $d(n)$ is the desired signal, $\mathrm{e}(\mathrm{n})$ is the error signal.

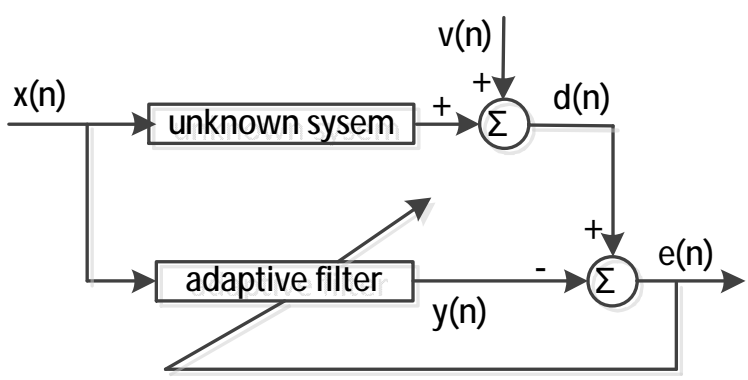

Fig. 1 The schematic diagram of adaptive filter

The objective function of LMS algorithm used the minimum mean square error which created of the output signal and desired signal ${ }^{[2]}$, the iterative formula of algorithm is:

$$
\begin{aligned}
& X(n)=\left[\begin{array}{lllll}
x(n) & x(n-1) & x(n-2) & \ldots & x(n-L-1)
\end{array}\right]^{T} . \\
& e(n)=d(n)-X(n)^{T} W(n)
\end{aligned}
$$




$$
\mathrm{W}(\mathrm{n}+1)=\mathrm{W}(\mathrm{n})+2 \mu \mathrm{e}(\mathrm{n}) \mathrm{X}(\mathrm{n})
$$

Among them: $\mathrm{W}(\mathrm{n})$ is the estimate value of weights vector of the filter; $\mathrm{L}$ is the order of the filter; $\mu$ is the step size factor, which used to control the stability and convergence rate of algorithm.

To ensure the convergence rate of LMS algorithm, the range of step size $\mu$ is: $0<\mu<1 / \lambda_{\max }, \quad \lambda_{\max }$ is the largest eigenvalue of the autocorrelation matrix of the input signal. From the above formulas we can see, the value of the step size plays an important role in the algorithm. when the step size is lager, the convergence rate is faster, but the steady-state error becomes larger; when the step size is lager, the convergence rate is faster, but the steady-state error becomes larger; when the step size is smaller, the steady-state error is smaller, but the convergence rate becomes slower. So, it is very difficult to overcome the contradiction between convergence rate and steady-state error in the fixed step size LMS algorithm. Therefore, many variable step size LMS algorithms are proposed.

\section{New Variable Step Size LMS Algorithm}

In order to find the optimal value of the weight coefficient, the variable step size LMS algorithm adjusts the step size factor in the process of algorithm convergence dynamically. When the weight coefficient is far from the optimal value, the step size factor should be larger to ensure the fast convergence rate. When the weight coefficient is close to the optimal value, the step size factor should be smaller to ensure a smaller steady-state error. Therefore, the step size factor changes with the error signal. According to the above adjustment principles, combining with the curve characteristics of the algorithm which proposed in reference [3], we proposed a new variable step size LMS algorithm based on cosine function. After translate the curve of cosine function, we get a new function of $\mathrm{y}=\cos (|\mathrm{x}|-\pi)+1$, and its curve is shown in figure 2 .

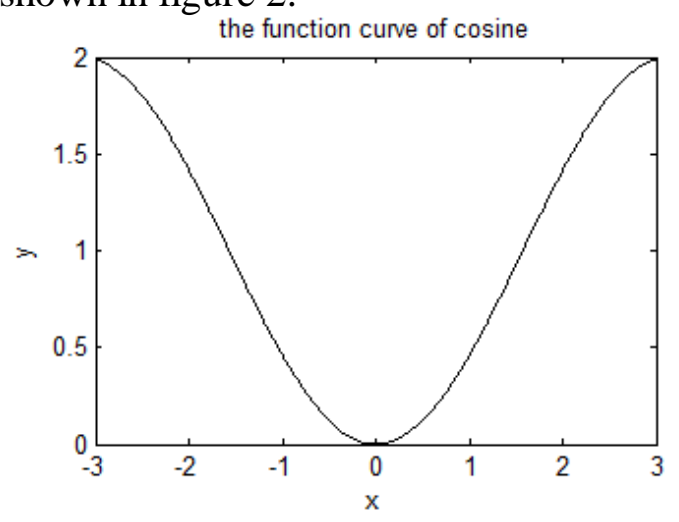

Fig. 2 The curve of $y=\cos (|x|-\pi)+1$

We can see from figure 2, when input signal $\mathrm{x}$ is far from zero, output signal y changes quickly, when input signal $x$ close to zero, $y$ changes slowly. In general, $x$ is more close to zero, $y$ changes more slowly, and the curve smooth at the bottom, which accord with the change rule of the variable step size factor and the error signal. Replace $\mathrm{x}$ with $\mathrm{e}(\mathrm{n})$, and $\mathrm{y}$ with $\mu(\mathrm{n})$, the new step size iterative formula is as follows:

$$
\mu(\mathrm{n})=\beta[\cos (\alpha|\mathrm{e}(\mathrm{n})|-\pi)+1] .
$$

Among them, parameter $\beta$ controls the value range of the curve, affects the convergence rate of the algorithm; parameter $\alpha$ controls the curve shape of error near zero. The function curve is shown in figure 3 when parameter $\beta=1, \alpha=1,2$ and 3. It can be seen that, with the increase of $\alpha, \mu(n)$ is increases, but when e(n) approaches to zero, $\alpha$ is larger, $\mu(n)$ changes greater, and the steady-state error becomes greater. Figure 4 is the function curve when parameter $\alpha=3, \beta=0.1,0.5$ and 1 . It can be seen that, the larger the value of $\beta$ is, the faster the convergence rate of curve. In order to ensure that the curve does not diverge, the value of $\beta$ should not be too large. 


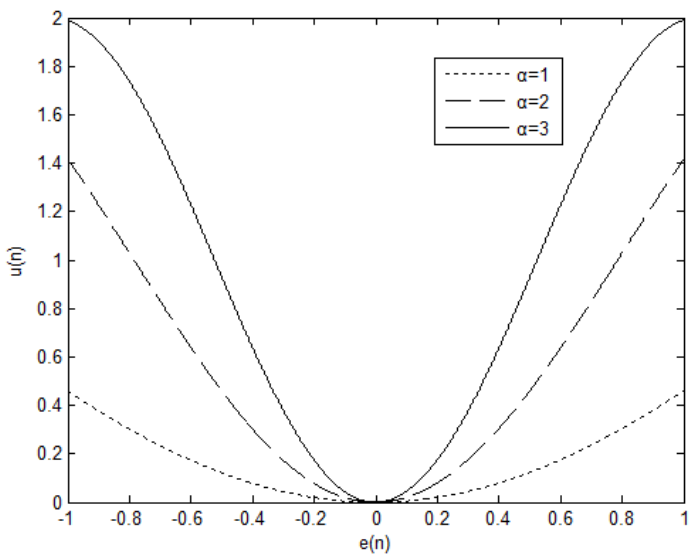

Fig. 3 The curve of $\mu(\mathrm{n})$ when $\beta=1, \alpha=1,2,3$

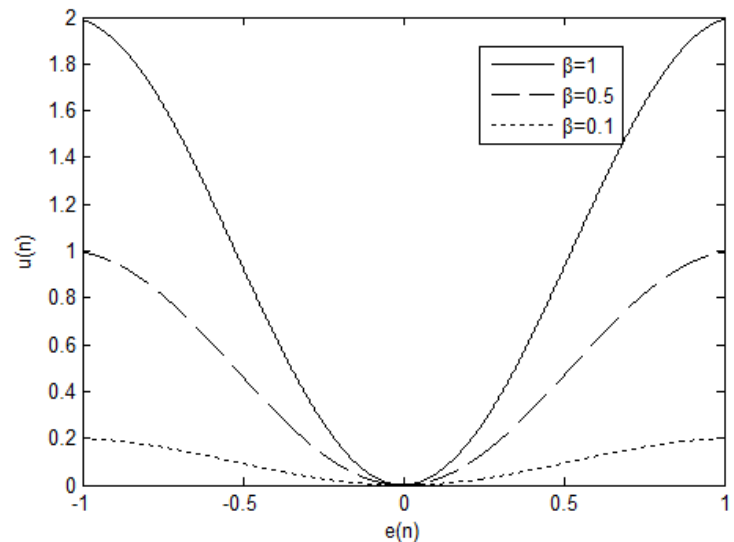

Fig. 4 The curve of $\mu(\mathrm{n})$ when $\alpha=1, \beta=0.1,0.5,1$

\section{Matlab Simulation}

Matlab software is used to simulate the adaptive noise cancellation system, and mainly compares the convergence rate and anti-interference of the three algorithms in different SNR. The three algorithms are fixed step size LMS algorithm, algorithm proposed in reference [4] and the new algorithm proposed in this paper. Simulation conditions: the input signal $\mathrm{x}(\mathrm{n})$ is a sinusoidal signal with amplitude is 1 , the noise signal $\mathrm{v}(\mathrm{n})$ is Gaussian white noise, the sampling point is 1000 , filter order is 2 . In order to get error curve accurately, we use the statistical mean of 500 simulations as the error curve. The algorithm parameters in this paper are set as: $\alpha=9.42, \delta=0.05$; the parameter of fixed step size LMS algorithm is set as $\mu=0.02$; the parameters of reference [4] are set as: $\alpha=0.05, \delta=0.01$.
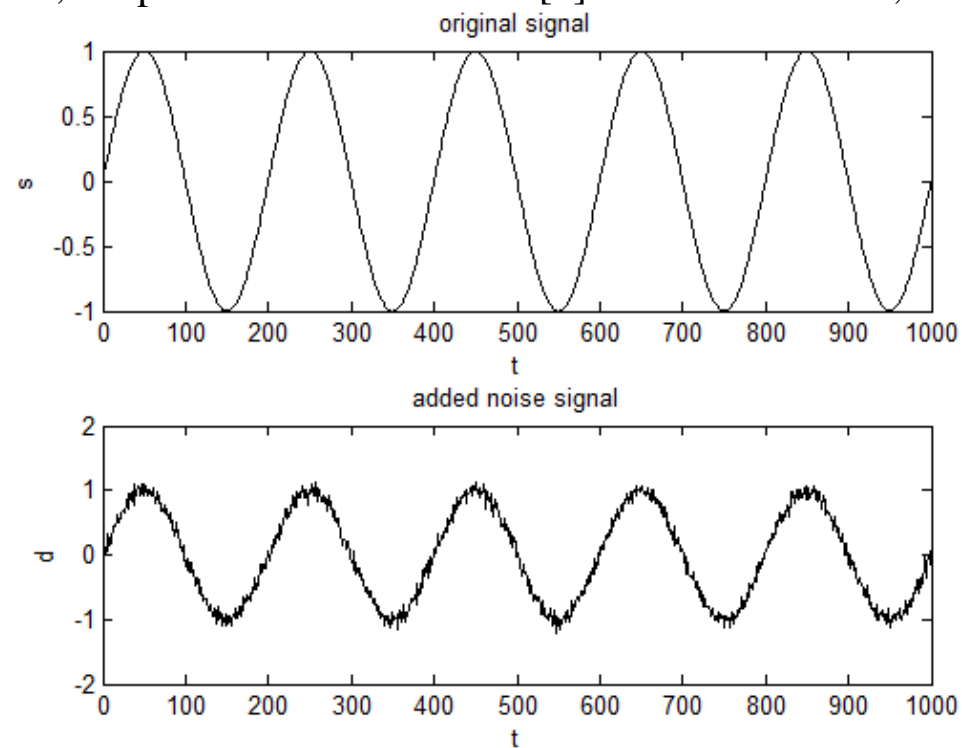

Fig. 5 The waveform of the original signal and the added noise signal 
In order to verify the convergence rate and anti-interference ability of the algorithm, the error curves of three algorithms are under different SNR conditions. Parameter setting is the same as above. As is shown in figure 6 , figure 7 , and figure 8 , the SNR of the error curve from top to bottom is $20 \mathrm{~dB}, 10 \mathrm{~dB}$ and $5 \mathrm{~dB}$.

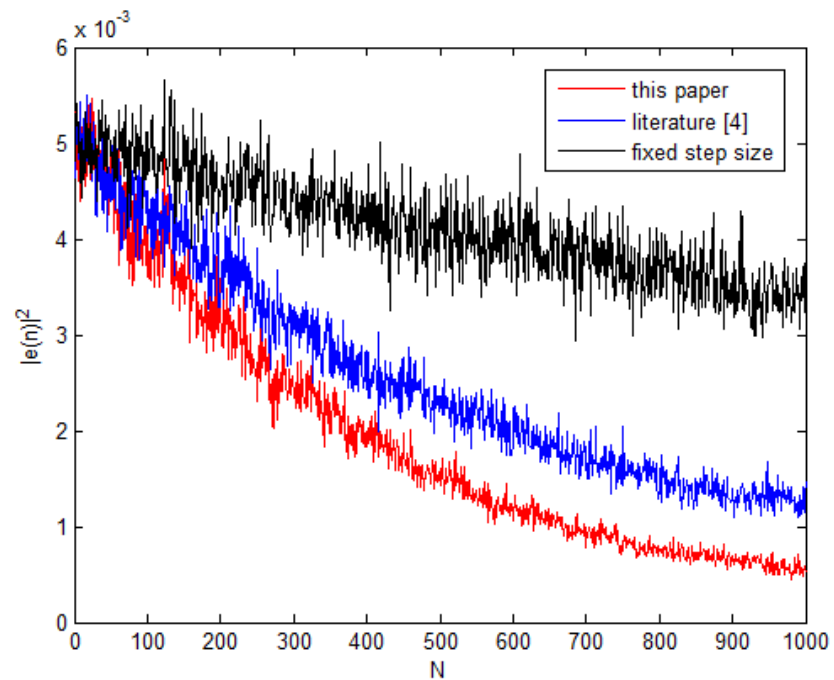

Fig. 6 The error curve of the three algorithms when SNR is 20

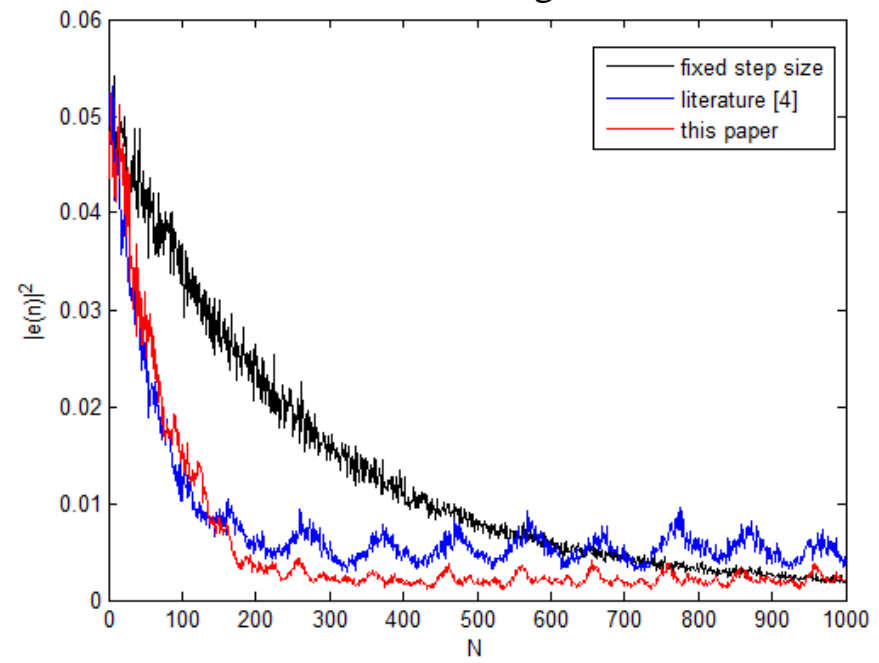

Fig. 7 The error curve of the three algorithms when SNR is 10

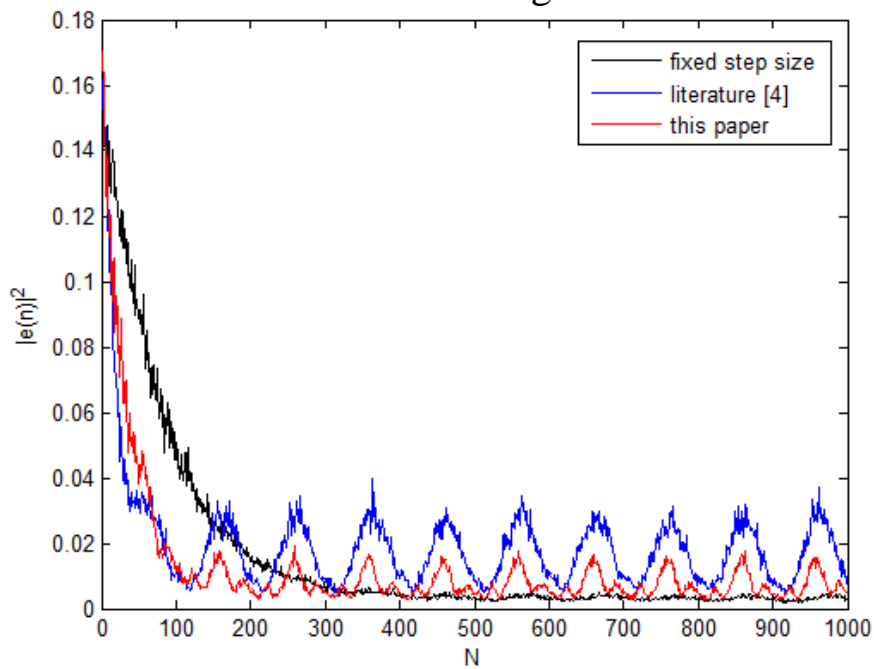

Fig. 8 The error curve of the three algorithms when SNR is 5

As is shown in figure 6, the SNR is larger, the mean square error of the algorithm in this paper is lower than the algorithm in the fixed step size and reference [4] significantly, it shows that the fast convergence rate and small steady-state error. As is shown in figure 8, the SNR is smaller, the 
algorithm in this paper and in reference [4] both reached the steady-state value at the 115th sampling point, and the steady-state error of the algorithm in this paper is lower than the algorithm in reference [4] significantly. The fixed step size algorithm has lower steady-state error because of its small step size, but it reached the steady-rate value at the 320th sampling points, which is slower than the algorithm in this paper. So we can conclude that the algorithm in this paper both ensure faster convergence rate and smaller steady-state error, and it is more suitable for signal denoising processing, especially for weak signal detection.

\section{Conclusions}

In this paper, through the mathematical transformation of the cosine function, we construct a new function of variable step size factor and error signal. The variable step size LMS algorithm on the premise of ensure the smaller amount of calculation, has fast convergence rate and small steady-state error, especially suitable for signal filtering and extraction under the condition of low SNR.

\section{References}

[1] YANG Yu and SHI Weilai: Study on adaptive filtering algorithm for variable step size LMS, submitted to Journal of Jiangsu institute of education(natural sciences), Vol. 27 (2011), p. 9-10.

[2] Paulo S.R.Diniz, in: Adaptive filtering: Algorithms and practical implementation fourth edition ( Electronic industry press Publishers, Brazil 2004).

[3] QIN Jingfan and OUYANG Jingzheng: A new variable step size of adaptive filtering algorithm. submitted to Journal of Data acquisition and processing, Vol. 12(1997), p. 171-194.

[4] ZHANG Jingjing, XU Shuai and ZHOU Feifei: A new variable step size LMS algorithm. submitted to Journal of Modern electronics technique, Vol. 37(2014), p. 11-13. 